Schweizerisches Jahrbuch für Entwicklungspolitik

27-2 | 2008

Migration und Entwicklung : Eine Zweckallianz

\title{
Migration und Entwicklung : eine Debatte zwischen Zwist und Annäherung
}

Alessandro Monsutti

\section{(2) OpenEdition}

1 Journals

Electronic version

URL: http://journals.openedition.org/sjep/306

DOI: 10.4000/sjep.306

ISSN: 1663-9677

Publisher

Institut de hautes études internationales et du développement

Printed version

Date of publication: 1 décembre 2008

Number of pages: $25-46$

ISBN: 978-2-940415-08-3

ISSN: $1660-5926$

\section{Electronic reference}

Alessandro Monsutti, « Migration und Entwicklung : eine Debatte zwischen Zwist und Annäherung », Schweizerisches Jahrbuch für Entwicklungspolitik [Online], 27-2 | 2008, Online erschienen am: 06

September 2010, abgerufen am 08 September 2020. URL : http://journals.openedition.org/sjep/306 ; DOI : https://doi.org/10.4000/sjep.306 


\title{
Migration und Entwicklung: eine Debatte zwischen Zwist und Annäherung
}

\author{
Alessandro Monsutti*
}

\section{Migration und Entwicklung: Wie viel Optimismus ist angebracht?}

Obwohl die Frage nach den Beziehungen zwischen Migration und Entwicklung (engl. migration-development nexus ${ }^{1}$ ) bereits seit dem Ende des Zweiten Weltkriegs für lebhafte Debatten sorgt, hat sie sich erst in den vergangenen Jahren einen festen Platz in Politik und Wissenschaft erobert. Ein Grund dafür ist das wachsende Interesse an den Rücküberweisungen der Migrantinnen und Migranten (remittances) als Instrument der Armutsbekämpfung und zur Förderung des Wirtschaftswachstums ${ }^{2}$. Seit den Anschlägen vom 11. September 2001 hat sich die Diskussion auf Fragen der internationalen Sicherheit ausgeweitet, wozu namentlich die Terrorismus- und Geldwäschereibekämpfung sowie die Rolle der Diasporen in Konflikten und in der Friedensförderung zählen.

Ein Beweis für das zunehmende Interesse internationaler Organe und staatlicher Stellen an Migrationsfragen war die Einsetzung der Global Commission on International Migration (GCIM) im Jahr 2003 in Genf. Ihr Auftrag lautete, den Rahmen für einen globalen und kohärenten Ansatz zur Bewältigung der Migrationsproblematik abzustecken. In ihrem Schlussbericht ${ }^{3}$ nannte die Kommission verschiedene Empfehlungen zur Koordinierung und Steuerung der Migrationsströme auf nationaler und internationaler Ebene. Der Bericht betonte, dass die Zahl der Migrantinnen und Migranten in den kommenden Jahren aus strukturellen Gründen (demografisches Gefälle, wirtschaftliche und politische Disparitäten usw.) weiter zunehmen dürfte. Die Staaten müssten dieser Feststellung Rechnung tragen und seien deshalb gefordert, pragmatische Politiken zu entwickeln. Dabei gelte es, dem Beitrag der Migrantinnen und Migranten an die Wirtschaft und die Gesellschaft der Gastländer zu würdigen und die Migrationsbewegungen zu nutzen, um eine gerechtere Entwicklung zu begünstigen, die Armut zu bekämpfen oder die Demokratisierung autoritärer

* Lehrbeauftragter am Institut de hautes études internationales et du développement (IHEID), Genf. Der Dank des Autors gilt Dawn Chatty und Gilbert Rist für ihre wertvollen Kommentare sowie Denise Efionayi-Mäder, Jean-Claude Huot, Gérard Perroulaz und Catherine Schümperli Younossian.

1 Vgl. hierzu die Sondernummer von International Migration, Bd. 40, Nr. 5, 2002, über die Beziehungen zwischen Migration und Entwicklung, insbesondere die einführenden Beiträge von N. NybergSørensen, N. Van Hear und P. Engberg-Pedersen, ,,The Migration-Development Nexus : Evidence and Policy Options - State-of-the-art Overview“, S. 3-47, und „The Migration-Development Nexus : Evidence and Policy Options", S. 49-73.

2 D. Kapur, Remittances: The New Development Mantra?, G-24 Discussion Paper Series, Nr. 29, New York; Geneva, United Nations Conference on Trade and Development, 2004.

3 Migration in an Interconnected World: New Directions for Action, Geneva, Global Commission on International Migration, October 2005. 
Regimes voranzutreiben. Vor diesem Hintergrund wurde im Herbst 2006 die 61. Tagung der UNO-Generalversammlung mit dem Hochrangigen Dialog über Internationale Migration und Entwicklung eröffnet. Die Durchführung dieser Gesprächsrunde, die ein Novum darstellte, zeugt davon, dass der Wille, das Phänomen der Migration zu verstehen und global zu bewältigen, auf der Agenda der Staatengemeinschaft einen festen Platz eingenommen hat.

Das heute ausgeprägte Streben nach Sicherheit wird paradoxerweise begleitet von einem zuweilen etwas naiven Optimismus in Bezug auf den Beitrag, den die Migrantinnen und Migranten zur wirtschaftlichen, politischen und gesellschaftlichen Entwicklung ihrer Herkunftsländer leisten können. Diese positive Sicht der Auswirkungen auf die Entwicklung der Länder des Südens, die durch zahlreiche in Auftrag gegebene Untersuchungen genährt wird und sich im politischen Diskurs mittlerweile etabliert hat, ist heute nicht nur bei der Weltbank ${ }^{4}$ und bei der Internationalen Organisation für Migration (IOM) ${ }^{5}$, sondern auch bei der Direktion für Entwicklung und Zusammenarbeit (DEZA) und bei anderen nationalen Entwicklungsagenturen ${ }^{6}$ vorherrschend, die die Migrationsproblematik in den Mittelpunkt ihrer Überlegungen gestellt haben. Mit frappierender Einhelligkeit erklären diese Einrichtungen, mit ihrer Tätigkeit die Risiken verringern $\mathrm{zu}$ wollen, die die Migrationsströme sowohl für die Migrantinnen und Migranten als auch für die Staaten bergen, und gleichzeitig die positiven Auswirkungen der internationalen Migration auf die Entwicklung der Länder des Südens zu unterstützen. Eine Win-win-Situation, in der sich die Interessen der Migrantinnen und Migranten aus den Entwicklungsländern und die Anliegen der Industriestaaten vereinbaren lassen, scheint in diesem institutionellen Diskurs in greifbare Nähe zu rücken.

Dieser Diskurs stützt sich auf die enormen Summen, die von Migrantinnen und Migranten in ihre Herkunftsländer überwiesen werden. Diese übersteigen das

4 Vgl. S.M. Maimbo and D. Ratha (eds.), Remittances: Development Impact and Future Prospects, Washington, DC, World Bank, 2005 (dieser Sammelband enthält unter anderem eine häufig zitierte Studie: D. Ratha, „Workers' Remittances: An Important and Stable Source of External Development Finance“, Global Development Finance 2003, Washington, DC, World Bank, 2003, S. 157-175). In Bezug auf Asien siehe Converting Migration Drains into Gains: Harnessing the Resources of Overseas Professionals, Manila, Asian Development Bank, 2006.

5 Die IOM hat massgeblichen Anteil an dieser Tendenz. Von der Vielzahl von Publikationen sei Folgende als Beispiel erwähnt: Migration for Development: Within and Beyond Frontiers, Geneva, International Organization for Migration, 2006. Sie versteht sich als Beitrag zum „, global effort to create measures that will maximize the potential development benefits of migration and mitigate its negative aspects" (<http://www.iom.int/jahia/Jahia/cache/offonce/pid/1674?entryId=10851>). Siehe auch N. Baruah, Remittances to Least Developed Countries (LDCs): Issues, Policies, Practices and Enhancing Development Impact, Geneva, International Organization for Migration, 2006; sowie B. Gosh, Migrants' Remittances and Development: Myth, Rhetoric and Realities, Geneva, International Organization for Migration; The Hague, The Hague Process on Refugees and Migration, 2006.

6 Siehe beispielsweise die Websites der US-amerikanischen, britischen, deutschen oder schweizerischen Entwicklungsagenturen: United States Agency for International Development (USAID), $<$ http://www.usaid.gov>; Department for International Development (DFID), <http://www.dfid.gov. uk/migration.asp>; Gesellschaft für Technische Zusammenarbeit (GTZ), <http://www.gtz.de/de/themen/ wirtschaft-beschaeftigung/15634.htm>; Direktion für Entwicklung und Zusammenarbeit (DEZA), $<$ http://www.deza.admin.ch/de/Home/Themen/Migration>. Das DFID hat insbesondere die Errichtung einer Plattform gefördert, auf der sich die Migrantinnen und Migranten über die zweckmässigsten Möglichkeiten zur Überweisung von Geld in ihre Herkunftsländer informieren können, <http:// www.sendmoneyhome.org >. 
Volumen der öffentlichen Entwicklungshilfe (APD) ${ }^{7}$ bei Weitem und dürften in den vergangenen Jahren weiter zugenommen haben (siehe Tabelle 1$)^{8}$.

Tabelle 1: Entwicklung der Rücküberweisungen von Migranten im Vergleich zur öffentlichen Entwicklungshilfe (APD), 2000-2006 (in Milliarden Dollar)

\begin{tabular}{lrrrrrrr}
\hline Nach Region & 2000 & 2001 & 2002 & 2003 & 2004 & 2005 & 2006 \\
\hline Ostasien und Pazifik & 17 & 20 & 20 & 35 & 39 & 45 & 47 \\
\hline Europa und Zentralasien & 13 & 13 & 14 & 17 & 23 & 31 & 32 \\
\hline Lateinamerika und Karibik & 20 & 24 & 28 & 35 & 41 & 48 & 53 \\
\hline Mittlerer Osten und Nordafrika & 13 & 15 & 16 & 20 & 23 & 24 & 25 \\
\hline Südasien & 17 & 19 & 24 & 31 & 31 & 36 & 41 \\
\hline Subsahara-Afrika & 5 & 5 & 5 & 6 & 8 & 9 & 9 \\
\hline Total Rücküberweisungen & 85 & 96 & 117 & 145 & 165 & 193 & 206 \\
\hline Öffentliche Entwicklungshilfe (APD) & 54 & 52 & 58 & 69 & 79 & 107 & 104 \\
\hline
\end{tabular}

Quelle der Angaben zu den Rücküberweisungen: World Bank, Global Development Finance 2007, Washington DC, World Bank, 2007, S. 54.

Quelle der Angaben zur APD: Organization for Economic Cooperation and Development, OECD.StatExtracts, <http://stats. oecd.org/wbos/Index.aspx?DatasetCode=ODA_DONOR> (konsultiert am 25. Juni 2008).

Dieser Optimismus birgt allerdings einen ersten Widerspruch: Die Migration wird zum Grundsatz ihrer Verneinung. Laut den internationalen Organisationen und den bilateralen Entwicklungsagenturen nämlich können Migrantinnen und Migranten zur Entwicklung ihrer Herkunftsländer beitragen; diese Entwicklung wiederum kann auf allgemeinerer Ebene mithelfen, die Migrationsströme einzudämmen. Die zu Beginn des 21. Jahrhunderts vorherrschenden politischen Diskurse legen einerseits den Schwerpunkt auf die Verringerung der internationalen Migration, gleichzeitig aber betonen sie, dass Rücküberweisungen die Armut und den gesellschaftlichen Ausschluss vermindern können. Dabei wird die Vision einer voluntaristischen, nicht institutionalisierten Entwicklung ,von unten nach oben“ quasi zum Glaubensgrundsatz erhoben. Da sie über mehr Mittel verfügen als die APD, wird den Migrantinnen und Migranten eine Rolle als potenzielle Investoren und mithin als wichtigste treibende Kraft der Entwicklung zugedacht ${ }^{9}$. Da stellt sich natürlich die Frage, ob diese etwas stereotype und redundante Orthodoxie nicht etwa die bi- und multilateralen Interventionen diskreditiert und dazu beiträgt, die Stärkung der sicherheitspolitischen Dispositive zu legitimieren. Migrationsbewältigung wäre damit nichts anderes als Ausdruck des Willens, die Migration zu kontrollieren und zu steuern ${ }^{10}$.

7 Allein die von Migranten über die offiziellen Kanäle überwiesenen Gelder - die nur etwa die Hälfte der informellen Rücküberweisungen ausmachen - dürften bis zu dreimal so hoch sein wie die öffentliche Entwicklungshilfe (Migration in an Interconnected World, op. cit., S. 26 und 85).

8 Zwischen 2001 und 2006 haben sich die über offizielle Kanäle abgewickelten Rücküberweisungen mehr als verdoppelt. Dieser Sachverhalt ist indessen auch auf die verstärkten staatlichen Kontrollen der Remittances seit den Anschlägen des 11. September 2001 zurückzuführen.

9 Vgl. beispielsweise den folgenden Bericht des britischen Unterhauses, dessen Titel bezeichnend ist: H. Harman, The Hidden Heroes of International Development: Remittances from the Southwark African Diaspora to Families and Villages in Africa, London, House of Commons, 2007.

10 Th. Faist, „Migrants as Transnational Development Agents: An Inquiry into the Newest Round of the Migration-Development Nexus“, Population, Space and Place, Bd. 14, 2008, S. 21-42.

Für eine schonungslose Kritik der Sicherheitsbestrebungen der Industrie- wie der Entwicklungsländer vgl. M.-C. Caloz-Tschopp, Les étrangers aux frontières de l'Europe et le spectre des camps, Paris, La Dispute, 2004 ; N. Bagayoko-Penone und B. Hours, Etats, ONG et production des normes sécuritaires dans les pays du Sud, Paris, L'Harmattan, 2006. 
Damit wird ein zweites Paradoxon erkennbar: Einerseits erkennt die neoliberale Theorie, welche heute in den internationalen Diskursen vorherrschend ist, dem Staat nur eine begrenzte Rolle zu, während Initiativen, welche von Individuen oder Körperschaften der Zivilgesellschaften ausgehen, eine grosse Bedeutung zugesprochen wird. Andererseits haben die Empfehlungen der Global Commission on International Migration zur Notwendigkeit einer verstärkten zwischenstaatlichen Koordination in der Realpolitik keinerlei Widerhall gefunden, denn die Staaten sind nicht gewillt, ihren souveränen Anspruch auf die Kontrolle der Ein- und Auswanderung in ihr Hoheitsgebiet preiszugeben, und bekunden Mühe, sich auf einen globalen Rahmen zur Bewältigung der Migrationsfrage zu einigen (die bestehenden Zusammenarbeitsvereinbarungen beziehen sich im Wesentlichen auf Sicherheitsfragen).

Die Bedeutung der heutigen Diskussionen für Theorie und Praxis sowie der von den internationalen Organisationen und gewissen Entwicklungshilfeagenturen geschürte Optimismus werden im vorliegenden Beitrag - wie auch in den übrigen Kapiteln dieses Beitrags - kritisch beleuchtet. Der während mehrerer Jahrzehnte zusammengetragene umfangreiche Korpus an empirischen Daten legt den Schluss nahe, dass ausgehend von den verschiedenen spezifischen Kontexten keine ",mechanische“ Verbindung zwischen Migration und Entwicklung oder zwischen Migration und Armutsverringerung abgeleitet werden kann. Die folgenden Feststellungen untermauern dies :

1. Migration und Entwicklung verhalten sich nicht umgekehrt proportional zueinander: Politische Ansätze, die darauf abzielen, „durch Entwicklung die Migration einzudämmen“ oder ,die Migration in den Dienst der Entwicklung zu stellen“, werden der Komplexität und der Verschiedenartigkeit der Situationen nicht gerecht.

2. Die vereinfachende Annahme, wonach Armut die Migration schürt, ist irreführend. In der Regel sind es nämlich nicht die Ärmsten, die sich zur Auswanderung entschliessen (diese Feststellung gilt mit gewissen Vorbehalten auch für Konfliktsituationen und Flüchtlinge).

3. Den höchsten Anteil an Personen, die in den Westen auswandern, weisen weder die Länder mit dem geringsten BIP noch jene mit dem höchsten Bevölkerungswachstum auf. $\mathrm{Zu}$ den wichtigsten Auswanderungsländern zählen heute Mexiko, Marokko, die Türkei oder auch die Philippinen - Länder also, die auf der weltweiten Reichtumsskala einen mittleren Rang belegen.

4. In den vergangenen Jahrzehnten wuchs die Zahl der Migrantinnen und Migranten in Europa (einschliesslich des ehemaligen Ostblocks), in Nordamerika und in Australien rascher als in anderen Regionen der Welt. Dennoch lässt sich die internationale Migration nicht auf eine massive Zuwanderung aus dem Süden in den Norden reduzieren. Ein bedeutender Anteil der weltweiten Migrantenbevölkerung - wovon eine grosse Mehrheit Flüchtlinge sind - lebt in Entwicklungsländern.

5. Der Optimismus in Bezug auf den Beitrag der Entwicklung zur Verringerung der internationalen Migrationsströme reproduziert den Mythos der Heimatverbundenheit der ländlichen Gemeinschaften: „Ein glücklicher Bauer bleibt auf seinem Land!“

6. Migrationen sind untrennbar mit der Geschichte der Menschheit verknüpft. Allerdings haben sie in der heutigen Zeit aufgrund neuer Kommunikationstech- 
nologien und verbesserter Transportmittel eine besondere Form angenommen. Migrantinnen und Migranten haben heute die Möglichkeit, die Kontakte unter ihresgleichen und mit den in der Heimat zurückgebliebenen Angehörigen zu pflegen und zu intensivieren. Aufgrund dieser Informationsflüsse verändern sich die Ansprüche der Menschen (neue Erwartungen, neue Möglichkeiten, neue Mittel und Wege...). Wie könnten die Menschen in diesem Kontext an ihrer Mobilität gehindert werden?

7. Die institutionellen Diskurse, die die Verbindung zwischen Migration und Entwicklung unterstreichen und auf die wichtige Rolle der Migrantinnen und Migranten für die Wirtschaft und die Gesellschaft ihrer Herkunftsländer hinweisen, zeigen viel versprechende Handlungsansätze auf, auch wenn diese die öffentliche Entwicklungshilfe nicht zu ersetzen vermögen. Allerdings stossen diese Ansätze in den Migrations- und Entwicklungshilfepolitiken der Länder der Organisation für wirtschaftliche Zusammenarbeit und Entwicklung (OECD) nur auf wenig Echo. Obwohl der Migrationsdimension grosse Beachtung geschenkt wird, wird sie kaum aus der offiziellen Rhetorik der internationalen Organisationen und der nationalen Entwicklungshilfeagenturen hinausgetragen und in den Entwicklungsprojekten selten adäquat berücksichtigt ${ }^{11}$. Zwar hat die Global Commission on International Migration empfohlen, das Management der Migrationsströme auf supranationaler Ebene zu verstärken, und gleichzeitig auf das Potenzial der Migrantinnen und Migranten für die Entwicklung ihrer Herkunftsländer und auf ihren Beitrag an den Wohlstand der Gastländer hingewiesen, aber die Regierungen halten nach wie vor an ihren angestammten Befugnissen in diesem Bereich fest.

\section{Ein Blick zurück: Situierung der Theorien im Laufe der Zeit}

Der heute dominierende Diskurs über den Beitrag der Migrantinnen und Migranten an die Entwicklung der Länder des Südens reflektiert wahrscheinlich die Dominanz des neoliberalen Paradigmas und der neoklassischen Wirtschaftstheorie. In jedem Fall aber lässt er Anzeichen einer gewissen Amnesie erkennen. Tatsächlich reichen die Anfänge der sozialwissenschaftlichen Debatte über die Beziehungen zwischen Migration und Entwicklung in die Nachkriegszeit zurück, als das System der internationalen Institutionen nach und nach Gestalt annahm. Deshalb erscheint es unverzichtbar, einen Blick zurück auf die Theorien zu werfen, die die vergangenen 60 Jahre geprägt haben und von sämtlichen Disziplinen - von der Ökonomie über die Anthropologie und die Politikwissenschaften bis zur Soziologie - aufgegriffen wurden.

Wie Hein de Haas treffend darlegt, durchlief die theoretische Debatte eine Pendelbewegung ${ }^{12}$. Die Meinungen schwankten zwischen dem Optimismus der

11 Als Beispiele sind die Entwicklungsprojekte der Fédération genevoise de coopération (FCG) zu nennen (siehe die kurze Analyse von Christelle Genoud und Yanik Marguerat in diesem Band). Zum Zeitpunkt der Herausgabe des vorliegenden Bandes hatte die Direktion für Entwicklung und Zusammenarbeit ihre konkrete Politik in diesem Bereich noch nicht abschliessend definiert (siehe Beitrag von Gérard Perroulaz im vorliegenden Band).

12 Siehe namentlich den folgenden Text, der die folgenden Ausführungen massgeblich geprägt hat: H. de Haas, Migration and Development: A Theoretical Perspective, Oxford, International Migration Institute, University of Oxford, 2008. Für eine allgemeinere Erörterung der Debatten zwischen den 
Modernisierungstheorie in den 1950er- und 1960er-Jahren und der von der Abhängigkeitstheorie inspirierten Skepsis der 1970er- und 1980er-Jahre. Diese beiden Sichtweisen stehen sich in Bezug auf die Rolle der Migrantinnen und Migranten diametral gegenüber, auch wenn diese Frage in der Entwicklungskontroverse nie explizit diskutiert wurde. Verstanden die Vertreter der einen Position die internationale Migration als Beitrag zur optimalen Ressourcenallokation und als notwendige Etappe in der Entwicklung der Dritten Welt, sahen die Anhänger der anderen darin ein Zeichen der Ungleichheit, die durch den Vormarsch des Kapitalismus weiter verschärft würde. Die Wende hin zu einer transnationalen Betrachtungsweise, die sich ab den 1990er-Jahren in der Migrationsforschung abzeichnete, führte zu einer nuancierteren Erörterung der Beziehungen zwischen Herkunfts- und Gaststaat und unterstrich, dass Migrantinnen und Migranten zahlreiche Beziehungen unterhalten und in mehreren Gesellschaften gleichzeitig als vollberechtigte Akteure auftreten können. Verschiedene migrationsökonomische Untersuchungen haben ausserdem gezeigt, dass die Entscheidung zur Auswanderung von Migranten und Nicht-Migranten gemeinsam getroffen wird und somit von der gesamten Haushaltsgemeinschaft getragen wird, die durch eine geografische Ausbreitung ihrer Mitglieder danach strebt, ihre Einkommensquellen zu diversifizieren und ihre Risiken zu vermindern. Parallel dazu trat die Dimension der internationalen Migration, die während langer Zeit von der Forschung wie auch von der Politik kaum angesprochen worden war, stärker zutage. Zwar hat sich der Anteil der Frauen unter den Migranten zwischen 1960 und heute nicht wesentlich erhöht ${ }^{13}$, aber die weltweiten ökonomischen Umwälzungen haben eine neue internationale Arbeitsteilung herbeigeführt. Das wirtschaftliche Gewicht der Frauen ausserhalb der Haushalte sowie ihre Rolle in der Entwicklung der Herkunftsländer sind heute weitgehend anerkannt ${ }^{14}$. Zahlreiche Frauen wandern aus eigenem Antrieb aus, verfolgen ein persönliches Lebensziel und bleiben dennoch häufig die Hauptversorgerinnen ihrer im Herkunftsland gebliebenen Familien ${ }^{15}$.

Chronologisch lässt sich die Debatte wie folgt situieren:

- 1950er- und 1960er-Jahre: Der durch die Modernisierungstheorie begründete Entwicklungsoptimismus herrscht vor. Migration gilt als Faktor für die Entwicklung der Drittweltländer.

- 1970er- und 1980er-Jahre: Die bisherige Betrachtungsweise wird abgelöst durch die Skepsis der Verfechter der Abhängigkeitstheorie und später der

verschiedenen entwicklungstheoretischen Strömungen siehe G. Rist, Le développement: histoire d'une croyance occidentale, Paris, Presses de Sciences Po, 2007 [3e édition], insbesondere S. 153 200 zur Modernisierungs- und Abhängigkeitstheorie.

13 F. Reysoo, „Féminisation de la migration“, in F. Reysoo et Ch. Verschuur (dir.), Femmes en mouvement: genre, migrations et nouvelle division internationale du travail, Berne, Commission suisse pour l'UNESCO; Direction du développement et de la coopération; Genève: Institut universitaire d'études du développement, 2004, S. 21.

14 Siehe z. B. B. Ehrenreich and A.R. Hochschild, The Global Women: Nannies, Maids and Sex Workers in the New Economy, New York, Metropolitan Books, 2003; oder P. Pessar and S.J. Mahler, „Transnational Migration: Bringing Gender In“, International Migration Review, Bd. 37, Nr. 3, 2003, S. 812-846.

15 Besonders verbreitet ist diese Konstellation auf den Philippinen, wo der Anteil der Frauen unter den Migranten 80 Prozent beträgt. Vgl. hierzu Ch. Verschuur, „Un regard genré sur les migrations“, in F. Reysoo et Ch. Verschuur (dir.), op. cit., S. 13. Siehe auch den Beitrag von Patricia A. Sto. Tomas im vorliegenden Band. 
Anhänger der Weltsystemtheorie und der Neomarxisten. Migration wird als Ausdruck der weltweiten Dominationsverhältnisse betrachtet.

- 1990er-Jahre: In den Sozialwissenschaften beginnt eine Periode der diffusen Zweifel, die die postmoderne Bewegung und die Kritik an der ,grossen Erzählung" der Aufklärung ${ }^{16}$ aufgreift. Parallel dazu setzen sich - als Antwort auf die Neue Ökonomie der Arbeitsmigration (new economics of labor migration, NELM), auf die transnationale Wende sowie auf die verstärkte Berücksichtigung der Gender-Dimension in der Migrationsforschung - nuanciertere Betrachtungsweisen des Verhältnisses zwischen Migration und Entwicklung durch.

- 2000er-Jahre: Ein neuer, von den internationalen Institutionen und den Entwicklungshilfeagenturen ausgehender Optimismus gewinnt die Oberhand. Dieser strebt eine Aufwertung der Rolle der Migrantinnen und Migranten an, die mit ihren Rücküberweisungen zur Entwicklung der Länder des Südens beitragen.

\section{Der Optimismus der neoklassischen Ökonomie und der Modernisierungstheorie}

Die Modernisierungstheorie entstand in den 1960er-Jahren rund um die Werke von W.W. Rostow ${ }^{17}$. Sie beruft sich auf das neoklassische Wirtschaftsmodell, sprich auf eine Theorie des Gleichgewichts, welches eine optimale Ressourcenallokation sicherstellen soll. Entsprechend dieser Grundannahmen wird davon ausgegangen, dass Migranten aus einkommensschwachen Regionen in reichere Gebiete und von dicht besiedelten in weniger dicht bewohnte Regionen wandern.

Auf mikroökonomischer Ebene wird Migration als Ergebnis der rationellen Entscheidungen der Individuen betrachtet, die die erwarteten Kosten und Nutzen gegeneinander abwägen und danach streben, ihren eigenen Vorteil zu maximieren. Aus makroökonomischer Sicht wiederum gelten Migrationsbewegungen als Resultat des Angebots- bzw. Nachfragegefälles auf dem Arbeitsmarkt. Aus Gegenden mit niedrigen Löhnen und einem Überangebot an Arbeitskräften (ländliche Gegenden mit einer mechanisierten Landwirtschaft) wandern Arbeitssuchende in Regionen aus, in denen ein Bedarf an zusätzlichen Arbeitskräften besteht oder wo die Löhne höher sind (urbane Zentren und Industrieregionen). In anderen Worten: Es handelt sich um eine Bewegung von Arbeitskräften „from labor-abundant to labor-scarce countries" 18 . Dies führt mit der Zeit zu einer grösseren Verfügbarkeit von Arbeitskräften in den Zielländern und

16 Im Sinne der berühmten Formulierung von J.-F. Lyotard, La condition postmoderne, Paris, Minuit, 1979 [Das postmoderne Wissen (Hg. von Peter Engelmann), Wien, Passagen Verlag, 2006].

17 W.W. Rostow, The Stages of Economic Growth: A Non-Communist Manifesto, London; Cambridge, Cambridge University Press, 1960. Wie aus dem Untertitel deutlich wird, ging es dem Autor auch darum, eine Alternative zu dem von der Sowjetunion vertretenen Entwicklungsmodell zur Diskussion zu stellen (obwohl beide Ansätze von denselben evolutionistischen Annahmen einer aus verschiedenen Stadien bestehenden Menschheitsgeschichte ausgehen, die jede Gesellschaft durchlaufen muss; vgl. G. Rist, op. cit., S. 164-171).

18 D.S. Massey, J. Arango, G. Hugo, A. Kouaouci, A. Pellegrino and J.E. Taylor, „Theories of International Migration: A Review and Appraisal“, Population and Development Review, Bd. 19, Nr. 3, 1993 , S. 431-466, Zitat S. 433. 
im Gegenzug zu einer Verknappung des Arbeitskräfteangebots in den Herkunftsgebieten der Migranten. Theoretisch wäre in Letzteren dadurch ein Anstieg der Löhne zu erwarten.

Gemäss diesem Modell zirkulieren Kapital und Personen in entgegengesetzten Richtungen. Nationale und internationale Migration gelten als Katalysatoren gesellschaftlicher Veränderung und als unverzichtbare Etappe, die die traditionellen Gesellschaften - welche sich durch eine geringe Produktivität und einen beschränkten Einsatz natürlicher Ressourcen auszeichnen - durchlaufen müssen, um in den Genuss der Vorzüge der Moderne zu kommen. Damit wird beispielsweise die Landflucht als Bestandteil eines globalen Entwicklungsprozesses betrachtet, der die industrielle Produktion konsolidiert. Der Modernisierungstheorie zufolge wirkt sich die Migration langfristig positiv auf die Entwicklung der Herkunftsregionen aus.

Für die Kritiker dieses Ansatzes war es ein Leichtes, daran zu erinnern, dass in vielen Fällen trotz Urbanisierung und Landflucht die von der Modernisierungstheorie beschworene wirtschaftliche Entwicklung ausgeblieben ist ${ }^{19}$. Dieses Modernisierungsmodell trägt den strukturellen Sachzwängen (wie z. B. dem ungleichen Zugang zu Ressourcen) nicht Rechnung. Es geht von der Annahme aus, dass alle Individuen dieselben Entscheidungsmöglichkeiten besitzen, und lässt die Heterogenität der Gesellschaften ausser Acht. Menschen haben Ziele und handeln nicht ausschliesslich aufgrund von Kosten-Nutzen-Überlegungen. Ausserdem ist es weniger die Höhe der Löhne an sich, die Migranten anzieht, als das Lohngefälle. Im Widerspruch zu den Annahmen der Gleichgewichtstheorien wandern zahlreiche Migranten aus wenig besiedelten Gegenden in Regionen mit hoher Einwohnerdichte aus.

Diese Betrachtungsweise des Migrationsphänomens, die geprägt ist durch eine bipolare Sicht, in der sich Herkunfts- und Zielgesellschaften gegenüberstehen, geht einher mit einer Unterscheidung zwischen Push-Faktoren, welche zum Wegzug aus dem Herkunftsland motivieren, und Pull-Faktoren, die die Migranten in ein allfälliges Zielland locken. Dieser Erklärungsansatz, der sich trotz schwer erkennbarem theoretischem Fundament in der Migrationsforschung grosser Beliebtheit erfreute ${ }^{20}$, lehnt sich an die neoklassische ökonomische Lehre an. Auch er schlägt ein Gleichgewichtsmodell vor, das auf individuellen Entscheidungen beruht. So führe Bevölkerungswachstum in ländlichen Gegenden zu einer zunehmenden Ressourcenknappheit. Diese wirke als Push-Faktor, der zahlreiche Mitglieder der ländlichen Bevölkerung zum Wegzug motiviere. Desgleichen sei die Hoffnung auf höhere Löhne in industrialisierten Gebieten als Pull-Faktor zu betrachten, denn die Migrantinnen und Migranten strebten danach, durch eine Auswanderung in diese Regionen ihre Einkommenssituation zu verbessern.

19 Siehe beispielsweise M. Kearney, „From the Invisible Hand to Visible Feet: Anthropological Studies of Migration and Development“", Annual Review of Anthropology, Bd. 15, 1986, S. 334.

20 Obwohl bereits Petersen die Theorie der Push- und Pull-Faktoren kritisierte, weil sie von einer weder historisch noch psychologisch begründeten universellen Tendenz zur Sesshaftigkeit ausgehe, wurde diese mit einer anderen Terminologie von Lee aufgegriffen, der die in den Herkunfts- und Zielregionen wirkenden Faktoren zu formalisieren suchte. Vgl. W. Petersen, „A General Typology of Migration“, American Sociological Review, Bd. 23, Nr. 3, 1958, S. 256-266, und E.S. Lee, „A Theory of Migration“, Demography, Bd. 3, Nr. 1, 1966, S. 47-57. 
Dieses Modell scheint bestechend einfach. Allerdings vermischt es verschiedene Analyseebenen, insbesondere die individuelle (Sachverhalte, die die Individuen wahrnehmen und bei ihren Entscheidungen berücksichtigen) und die gesellschaftliche (z. B. die Arbeitsmarktstruktur). Um es mit den Worten de Haas' auszudrücken: „The push-pull model is a static model focusing on external factors that cause" migration that is unable to analytically situate migration as an integral part of broader transformation processes, and therefore seems of limited analytical use. " ${ }^{21}$ Ausserdem vernachlässigt das Push-Pull-Modell die Tatsache, dass innerhalb ein und derselben Bevölkerungsgruppe sowohl Auswanderungs- als auch Einwanderungsbewegungen stattfinden. Wie liesse sich sonst erklären, dass zahlreiche Migranten in ihre Heimat zurückkehren, obwohl sich die Push- und Pull-Faktoren nicht zwangsläufig verändert haben? Es handelt sich somit um ein eher deskriptives als analytisches Modell, in dem Migration nicht als Prozess betrachtet wird. Es rückt die Ursachen der Migration in den Brennpunkt der Überlegungen, vernachlässigt dabei aber die von Migrationsbewegungen hervorgerufenen Veränderungen.

\section{Die Kritik der dependantistas}

Als Antwort auf die oben erläuterte Sichtweise kristallisierten sich gegen Ende der 1960er-Jahre pessimistischere Visionen der Entwicklung - und mithin der Beziehungen zwischen Migration und Entwicklung - heraus. Insbesondere die sogenannte Dependenzschule übte in quasi sämtlichen Punkten Kritik an der Modernisierungstheorie. Ein wichtiger Bezugspunkt dieser Schule war das Werk des Wirtschaftshistorikers Andre Gunder Frank, der die Wendung „Entwicklung der Unterentwicklung“ prägte. Sie fand unter anderem in einer Gruppe südamerikanischer Intellektueller - der dependantistas - eine treue Anhängerschaft. Dieser Gruppe gehörte auch der brasilianische Soziologe Fernando Henrique Cardoso an, der von 1995 bis 2003 als Staatspräsident Brasiliens amtierte $^{22}$. Verstärkung erhielt diese Gruppe von den Anhängern der Weltsystemtheorie und den Neomarxisten ${ }^{23}$, die sich ebenfalls der Modernisierungsschule entgegensetzten.

Diese Autoren berufen sich auf die Feststellung, dass die politische und wirtschaftliche Macht ungleich zwischen entwickelten und unterentwickelten Ländern verteilt ist. Die verschiedenen Gruppen der Weltbevölkerung hätten nicht alle den gleichen Zugang zu Ressourcen. Anstatt diese Ungleichheiten einzuebnen, verstärke der sich ausbreitende Kapitalismus diese weiter zugunsten des Zentrums und auf Kosten der Peripherie. Das Weltsystem sei von Ausbeutungsmechanismen geprägt: Natürliche und menschliche Ressourcen aus dem Süden dienten als Nahrung für das Wachstum des Nordens. So wird beispielsweise der

21 H. de Haas, op. cit., S. 11.

22 A.G. Frank, „The Development of Underdevelopment“, Monthly Review, Bd. 18, Nr. 4, 1966, S. 17-31; F.H. Cardoso, Les idées à leur place: le concept de développement en Amérique latine, Paris, A.-M. Métailié, 1984.

23 I. Wallerstein, The Modern World-System I: Capitalist Agriculture and the Origins of the European World-Economy in the Sixteenth Century, New York, Academic Press, 1974; The Modern WorldSystem II: Mercantilism and the Consolidation of the European World-Economy, 1600-1750, New York, Academic Press, 1980; The Modern World-System III: The Second Era of Great Expansion of the Capitalist World-Economy, 1730-1840s, New York, Academic Press, 1989. 
Grundsatz des Freihandels als Prozess beschrieben, der tendenziell die Begüterten begünstigt und die Anstrengungen der Benachteiligten zunichte macht. Dies bewirkt eine Verschärfung der Asymmetrie anstatt eine schrittweise - wenn auch zuweilen schmerzhafte - Annäherung an ein Gleichgewicht. Um dieser Entwicklung Einhalt zu gebieten, müsse das in den westlichen Ländern vorherrschende Aneignungs- und Anhäufungsregime reformiert werden. $\mathrm{Zu}$ diesem Zweck sei die systematische Nutzung natürlicher und menschlicher Ressourcen der Peripherie durch das Zentrum zu unterbinden, indem den Staaten eine stärkere regulierende Rolle zugedacht und die Industrialisierung der Drittweltländer gefördert wird, damit diese sich ebenfalls entwickeln können.

In diesem konzeptuellen Rahmen erscheint die internationale Migration als eine der zahlreichen Facetten der weltweiten Dominationsverhältnisse. Sie schaden der Entwicklung des Südens, vertiefen die Kluft zwischen Zentrum und Peripherie und führen zu einem Auseinanderbrechen der Solidaritätsbeziehungen in den Herkunftsgesellschaften der Migranten. Im Gegensatz zu den Annahmen der oben erörterten Theorien zirkulieren Migrationsströme nicht in entgegengesetzter Richtung zum Kapital, sondern folgen diesem. Die Einzelnen sind in ihren Entscheidungen nicht frei, sondern orientieren sich an den strukturellen Zwängen, die in den Mittelpunkt der Analysen gestellt werden müssen. Migrationsströme tragen demnach dazu bei, die Probleme der Unterentwicklung und der Abhängigkeit der Drittweltländer zu verstärken, denn für Letztere bewirkt die Flucht vor dem Elend einen Abfluss von Humankapital und somit ein Ausbluten der Gesellschaft ${ }^{24}$.

Diesem Ansatz wurde vorgeworfen, deterministisch und zu verallgemeinernd zu sein, um adäquate Forschungsfragen zu spezifischen Gebieten zu liefern. Er vernachlässigt die von der Peripherie an den Tag gelegten Widerstands- und Wiederaneignungsstrategien. Tatsächlich stützt er sich auf eine vereinfachende Sicht der internationalen Arbeitsteilung und geht von einem einzigen weltweiten Zentrum aus, wohingegen der multizentrische Charakter heute offenkundig ist. Ausserdem reduziert er die Menschen auf ihre Rolle als Opfer der strukturellen Kräfte und erkennt ihnen jegliche Handlungsmöglichkeiten ab. Kulturelle Faktoren werden vernachlässigt, während die Bedeutung der Wirtschaftsfragen gegenüber jener der politischen Beziehungen überschätzt wird ${ }^{25}$. Die dependantistas prangerten die Mechanismen an, die Unterentwicklung verursachen, und forderten den Abbau der strukturellen und historischen Barrieren, die die Dritte Welt in ihrem Untertanenstatus gefangen hielten. Dabei gelang es ihnen allerdings nicht, die vorherrschende Auffassung von Entwicklung als Versprechen auf eine bessere Welt für alle zu hinterfragen ${ }^{26}$.

24 Diese negative Sichtweise der Süd-Nord-Migration ist auch heute noch unter afrikanischen Intellektuellen weit verbreitet. Viele von ihnen sind überzeugt, die massive Auswanderung nach Europa sei das Ergebnis der Dominierung und der erneuten Kolonisierung unter dem Banner der liberalen Globalisierung, welche zu einer Verarmung und zum Ausschluss weiter Teile der Weltbevölkerung führt. Sie rufen die Bevölkerung Afrikas auf, ihr Schicksal und die Entwicklung ihres Kontinents selbst in die Hand zu nehmen. Siehe unter anderem Aminata Traoré, L'Afrique humiliée, Paris, Fayard, 2008, oder Jean-Emmanuel Pondi (dir.), Immigration et diaspora: un regard africain, Paris; Yaoundé, Maisonneuve et Larose, 2007.

25 M. Kearney, op. cit., S. 331-361.

26 G. Rist, op. cit., S. 194-195. 
Dieser kurze Abriss zeigt, dass die Gegenüberstellung monoparadigmatischer Visionen - sei es in Bezug auf die Modernisierung oder im Hinblick auf die Abhängigkeit - überwunden werden muss. Die Beziehungen zwischen Migration und Entwicklung sind komplexer und weniger geradlinig, als diese klassischen Positionen oder der heute vorherrschende institutionelle Optimismus vermuten lassen. Es gibt keine einfachen Antworten, denn zwischen diesen beiden Phänomenen - wie auch zwischen Migration und Armut - besteht keine automatische, mechanische oder anderweitige Verbindung. Gefragt ist ein pluralistischer und flexibler Ansatz, der die zeitliche und räumliche Verschiedenartigkeit der Konstellationen abdeckt und die Beziehungen zwischen Migration und Entwicklung umfassend betrachtet ${ }^{27}$.

Tatsächlich dürfte die heute auf politischer Ebene beobachtete Aufwertung der Rolle der Migranten dazu beitragen, dass Ursachen und Folgen der Migration voneinander getrennt untersucht werden, und dem Gedanken Vorschub leisten, die Migrationsströme liessen sich durch die Bewältigung der Probleme in den Herkunftsländern eindämmen. Dabei gerät in Vergessenheit, dass Migrationsprozesse eine eigenständige Dynamik aufweisen, dass sie sich relativ selbstständig reproduzieren können und dass sie sich weder auf rein ökonomische Faktoren noch auf eine Aggregierung individueller Entscheidungen reduzieren lassen. Ausserdem sind die strukturellen Auslöser für Migrationen sowohl in den Zielländern (Arbeitsmarkt, demografische Ungleichgewichte usw.) als auch in den Herkunftsländern (Armut, Unterentwicklung usw.) angesiedelt.

Für eine mehrschichtige Betrachtungsweise der Beziehungen zwischen Migration und Entwicklung spricht aber auch die demografische Transition in Bevölkerungsgruppen, deren Lebensbedingungen sich verbessern. Ist die Anfangsphase noch geprägt von hohen Geburten- und Sterblichkeitsraten und gleichzeitig geringem Bevölkerungswachstum, so bewirken eine Verbesserung der sanitären Bedingungen und der Ernährungslage sowie ein breiterer Zugang zu Schulen sehr rasch ein Absinken der Sterblichkeit, während sich die Geburtenrate zunächst nur unwesentlich verändert. Dies führt zu einem raschen Bevölkerungswachstum und zu einem Bevölkerungsüberschuss, mit dem die Produktivitätssteigerungen in der Landwirtschaft und die Schaffung von Arbeitsplätzen im sekundären und im aufstrebenden tertiären Sektor nicht immer Schritt zu halten vermögen. Ein solches Ungleichgewicht herrschte im Europa des 19. Jahrhunderts und konnte nur durch die Auswanderung von zig Millionen Menschen in die Neue Welt entschärft werden. Ganz offensichtlich diente die Auswanderung damals als Ventil für das Bevölkerungswachstum. In einer späteren Phase nimmt die Sterblichkeit weiter wenn auch langsamer - ab, und auch die Geburtenraten gehen spürbar zurück, bis sich beide Kurven auf einem tieferen Niveau als zuvor stabilisieren. Danach sinkt

27 Eine kritische Präsentation der Literatur findet sich in folgenden Werken von H. de Haas : Engaging Diasporas: How Governments and Development Agencies Can Support Involvement in the Development of Origin Countries, Oxford, International Migration Institute, University of Oxford, 2006; Turning the Tide? Why „Development instead of Migration“ Policies are Bound to Fail, Oxford, International Migration Institute, University of Oxford, 2006; Remittances, Migration and Social Development: A Conceptual Review of the Literature, Geneva, United Nations Research Institute for Social Development, 2007 ; Migration and Development, op. cit. 
das Bevölkerungswachstum weiter und erreicht zuweilen gar negative Werte, wie es heute in den westlichen Ländern der Fall ist ${ }^{28}$. Die Länder des Südens befinden sich heute mitten in dieser demografischen Transition, haben jedoch nicht dieselben Auswanderungsmöglichkeiten wie Europa in der Zeit zwischen den Napoleonischen Kriegen und dem Ersten Weltkrieg.

Der für die Transition bezeichnende Bevölkerungsdruck, den in der Anfangsphase des wirtschaftlichen Wachstums in diesen Regionen herrscht, ist indessen nicht der einzige Grund für die zunehmende Auswanderung. Dank des wirtschaftlichen Aufschwungs haben die Menschen mehr Mittel zur Verfügung, um eine Auswanderung zu finanzieren. Erst bei einem höheren Entwicklungsstand dürfte der Strom der Emigranten nachlassen. Dieses Phänomen wird in der englischsprachigen Literatur als migration hump bezeichnet. Tatsächlich wurde beobachtet, dass sich nicht nur die Zahl der Migranten deutlich verringert, sondern dass sich auch die Migrationslaufbahnen diversifizieren, sobald die Migranten und die Bevölkerung in ihren Herkunftsländern über mehr finanzielle Ressourcen verfügen.

\section{Abbildung 1: Auswirkungen der Entwicklung auf die Migrationsströme : der migration hump}
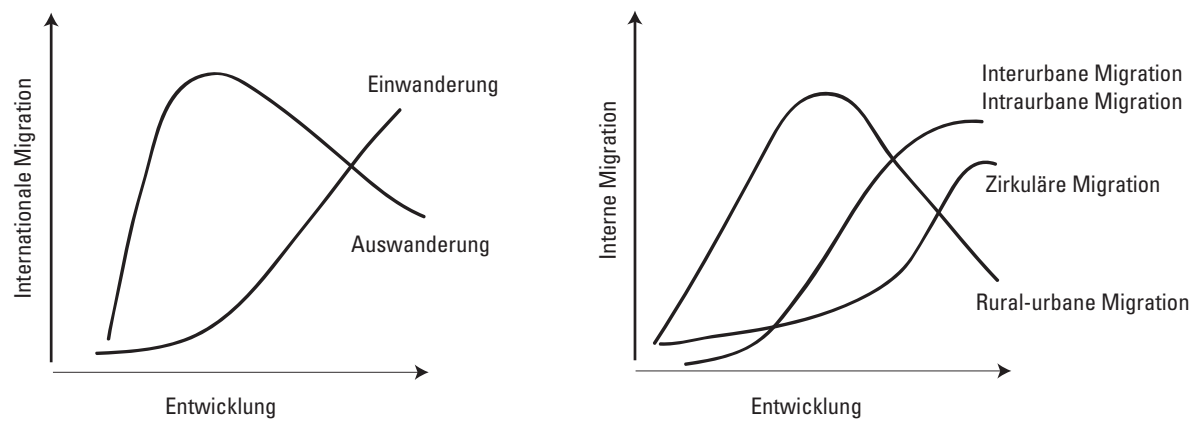

Quelle: Hein de Haas, Migration and Development: A Theoretical Perspective, Oxford, University of Oxford, 2007, S. 18.

Offensichtlich verhalten sich Migration und Entwicklung nicht umgekehrt proportional zueinander, und auch zwischen Migration und Armut ist kein eindeutiger Bezug erkennbar. Ausschlaggebend ist der jeweilige Kontext, der abhängig von der Region wie auch zeitlich stark variieren kann. Ausserdem ist die Beziehung umkehrbar: Bei Entwicklungsrückschritten oder in Konfliktsituationen können Einwanderungsländer zu Auswanderungsländern werden, wie die Beispiele Elfenbeinküste und Demokratische Republik Kongo zeigen. Die Kausalbeziehung zwischen den beiden Kategorien von Phänomenen steht somit nicht fest. Tatsächlich führen die unter dem Begriff „Entwicklung“ zusammengefassten sozialen und ökonomischen Veränderungen tendenziell in einer ersten Phase dazu, dass mehr Menschen die Möglichkeit zum Auswandern erhalten. Langfristig ist eine Diversifizierung der Migrationsmodelle erkennbar ${ }^{29}$.

28 In den Industrieländern sind 56 Prozent des Bevölkerungswachstums zwischen 1990 und 2000 auf die internationale Migration zurückzuführen. Für Europa liegt dieser Anteil gar bei 89 Prozent, und für Nordamerika für die Zeit zwischen 1995 und 2000 bei 75 Prozent. Vgl. hierzu Migration in an Interconnected World, op. cit., S. 84-85.

29 H. de Haas, Migration and Development, op. cit., S. 17. 
Diese Überlegungen dürften Anlass genug sein, um gewisse Gemeinplätze zu hinterfragen, so etwa jenen, wonach die nach standardisierten und allgemeingültigen Kriterien gemessene Armut die Hauptursache für die Süd-Nord-Migration sei. Weitaus entscheidender ist das Armutsgefälle, das heisst die relative Armut. Eine wichtige Rolle spielen zudem weitere Faktoren wie die Einschulungsrate oder die Lebenserwartung. Offensichtlich sind die Auswirkungen der Entwicklung auf die Migration differenziert zu bewerten. Eine weniger in Ideologien verhaftete, für regionale Unterschiede empfängliche Perspektive bietet einen Ausweg aus der an Ort und Stelle tretenden Debatte zwischen Optimisten und Pessimisten. Damit es aber gelingt, den nötigen Abstand zu wahren gegenüber den auf politischer und institutioneller Ebene dominierenden Diskursen, braucht es einen theoretischen und analytischen Rahmen, der ausreichend flexibel ist, um Variationen zu erklären, sowie eine umfassende empirische Forschung, die das gesamte Spektrum der möglichen Konstellationen abdeckt. Dabei müssen die Initiativen der Migranten und Nicht-Migranten (ihr Handlungsspielraum, um die Entwicklung des strukturellen und institutionellen Rahmens ihrer Existenz voranzutreiben), aber auch die strukturellen Sachzwänge, unter denen sie leben (Markt, globale und lokale Machtverhältnisse usw.), gleichermassen berücksichtigt werden.

Ein solches Forschungsprogramm erfordert eine Abkehr von der Betrachtung der Herkunfts- beziehungsweise der Zielländer der Migrantinnen und Migranten und eine Hinwendung zu einer Analyse der transversalen Dimension der Migrationssysteme. In der Tat sind gewisse Gebiete durch Austauschsysteme miteinander verbunden, die es ermöglichen, einige spezifische, wenn auch poröse Migrationseinheiten zu identifizieren:

$\checkmark$ das amerikanische System, in dem Mexiko als Drehscheibe und gleichzeitig Herkunfts-, Transit- und Zielland die Vereinigten Staaten und Kanada mit Lateinamerika verbindet;

$\square$ das euro-mediterrane System mit zwei grossen Migrationsachsen, nämlich jene von Subsahara-Afrika über Marokko und Tunesien und jene vom Mittleren Osten über die Türkei und den Balkan nach Europa ${ }^{30}$;

$\checkmark$ das System mit den Anrainerstaaten des Persischen Golfs als Zentrum, die einen bedeutenden Zustrom von Migranten aus dem übrigen Nahen Osten, aus dem indischen Subkontinent und aus Südostasien verzeichnen;

$\checkmark$ das System, das alle asiatischen Länder via Südostasien mit Australien und Japan verbindet.

Für die Betrachtung dieser Einheiten als Systeme erscheint es sinnvoll, die Lage in den Herkunfts- und Transitländern vor dem Hintergrund der Situation in den Zielländern zu betrachten und umgekehrt, denn die verschiedenen Etappen einer Migrationsroute sind miteinander verbunden. Parallel lassen sich durch die gleichzeitige Betrachtung von Ursachen und Konsequenzen dieser Ströme und der Beziehungen zwischen Migranten und Nicht-Migranten, Herkunftsgebieten und Zielländern die globalen Migrations- und Entwicklungskontexte herausarbeiten.

30 Siehe hierzu das unlängst erschienene Werk von A. Tarrius, La remontée des Sud: Afghans et Marocains en Europe méridionale, La Tour-d'Aigues, Editions de l'Aube, 2007. 


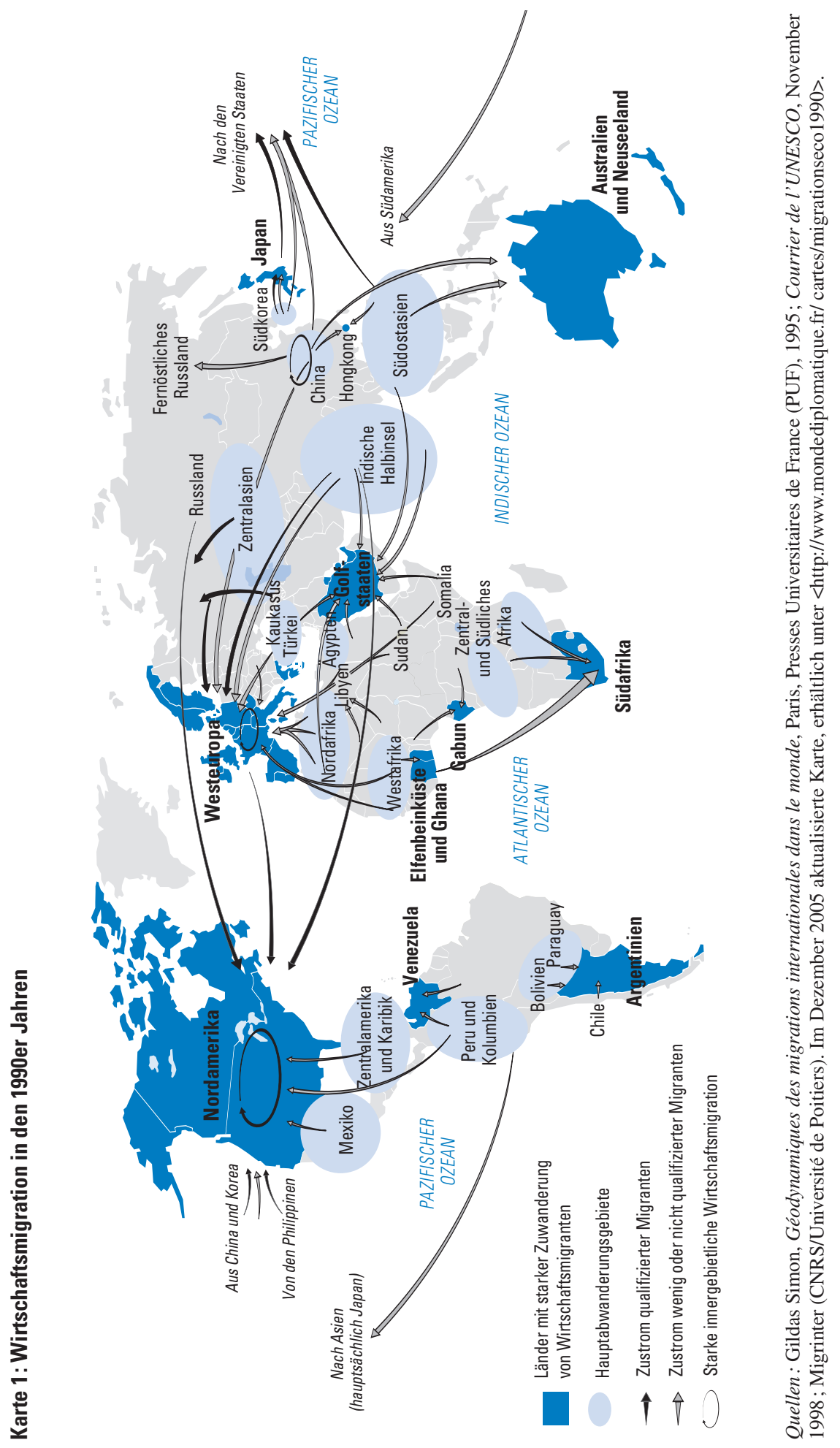


Aber auch abseits der Entwicklungsdebatte hat die Migrationsforschung seit Mitte der 1980er-Jahre vor dem Hintergrund der Hinterfragung und Dekonstruktion, die die Sozialwissenschaften zu dieser Zeit prägten, einen beeindruckenden Aufschwung durchlaufen. Immer häufiger wird die Mobilität der Menschen nicht mehr als unidirektionale, von einer mehr oder weniger erfolgreichen Integration der Migranten gekrönte Wanderung von A nach B, sondern als komplexes Phänomen eines andauernden Kreislaufs verstanden ${ }^{31}$. Personen, die über staatliche, kulturelle und geografische Grenzen hinweg Mehrfachbeziehungen entwickeln und pflegen (seien diese nun familiärer, wirtschaftlicher, gesellschaftlicher, religiöser oder politischer Natur), werden als sogenannte Transmigranten bezeichnet. Es handelt sich dabei um Personen, die eine Vielzahl von Beziehungen zwischen mehreren Orten pflegen und deren gesellschaftliches Leben in Netzen stattfindet, die sich über mehrere Nationalstaaten erstrecken ${ }^{32}$.

Gemäss dieser heute als Transnationalismus bezeichneten Perspektive sind soziokulturelle Gruppen nicht mehr als voneinander getrennte und territorial eingegrenzte Einheiten zu verstehen. Durch die Überbrückung der Gegenüberstellung von Herkunfts- und Zielgesellschaft erlaubt dieser Ansatz auch die Überwindung der formalen Kategorisierung in permanente, befristete, erzwungene und freiwillige Migration und ermöglicht es ferner, die Integration im Zielland oder die Rückkehr in die Heimat nicht mehr als Endpunkt des Migrationszyklus zu betrachten. Das Konzept der transnationalen Netze dient einer ganzen neuen Wissenschaftlergeneration zur Erforschung der Zwangsmigration ${ }^{33}$. Diese

31 Für eine umfassende Präsentation und Beurteilung dieser Neuorientierung siehe S. Vertovec, „Conceiving and Researching Transnationalism“, Ethnic and Racial Studies, Bd. 22, Nr. 2, 1999, S. 447-462. Von den zahlreichen neueren Werken zu dieser Frage seien Folgende erwähnt: N. Al-Ali and Kh. Koser (eds.), New Approaches to Migration? Transnational Communities and the Transformation of Home, London; New York, Routledge, 2002; Th. Faist, The Volume and Dynamics of International Migration and Transnational Social Spaces, Oxford, Oxford University Press, 2000.

Das der Integration zugrunde liegende klassische Migrationsmodell, auf das sich die nationalen Politiken mehrheitlich berufen, aber auch gewisse Auffassungen von Staatsbürgerlichkeit und Nationalstaat werden durch diese Betrachtungsweise in Frage gestellt. Siehe Th. Faist and P. Kivisto (eds.), Dual Citizenship in a Global Perspective: From Unitary to Multiple Citizenship, New York, Palgrave Macmillan, 2007.

32 N. Glick Schiller, L. Basch and C. Blanc-Szanton (eds.), Towards a Transnational Perspective on Migration: Race, Class, Ethnicity, and Nationalism Reconsidered, New York, The New York Academy of Sciences, 1992; sowie von denselben Autoren „From Immigrant to Transmigrant: Theorizing Transnational Migration“, Anthropological Quarterly, Bd. 68, Nr. 1, 1995, S. 48-63.

33 Stellvertretend für die umfangreiche Literatur hierzu seien folgende Werke erwähnt: N. Al-Ali, R. Black and Kh. Koser, „Refugees and Transnationalism: The Experience of Bosnians and Eritreans in Europe“, Journal of Ethnic and Migration Studies, Bd. 27, Nr. 4, 2001, S. 615-634; G. Chatelard, Iraqi Forced Migrants in Jordan: Conditions, Religious Networks, and the Smuggling Process, Working Papers, Nr. 49, Florence, Robert Schuman Center for Advanced Studies, European University Institute, 2002; C. Horst, Transnational Nomads: How Somalis Cope with Refugee Life in the Dadaab Camps of Kenya; New York, Oxford, Berghahn Books, 2006; E. Marx, „The Social World of Refugees A Conceptual Framework“, Journal of Refugee Studies, Bd. 3, Nr. 3, 1990, S. 189-203; A. Monsutti, Guerres et migrations: réseaux sociaux et stratégies économiques des Hazaras d'Afghanistan, Neuchâtel, Institut d'ethnologie; Paris, Maison des sciences de l'homme, 2004, sowie „Afghan Migratory Strategies and the Three Solutions to the Refugee Problem“, Refugee Survey Quarterly, Bd. 27, Nr. 1, 2008, S. 58-73; S. Shami, „Transnationalism and Refugee Studies: Rethinking Forced Migration and Identity in the Middle East", Journal of Refugee Studies, Bd. 9, Nr. 1, 1996, S. 3-26. 
Autoren lehnen sich an die theoretischen und methodischen Überlegungen an, die primär zur Erforschung der freiwilligen Migration entwickelt wurden, ohne dabei jedoch die besondere rechtliche Stellung der Flüchtlinge und die ihnen drohenden Gefahren zu leugnen. Verschiedene Feststellungen rechtfertigen dieses Vorgehen: Zum einen sind als Flüchtlinge anerkannte Personen nicht nur Opfer eines übermächtigen Schicksals, sondern auch Akteure, die auf schwierige Umstände reagieren und sich dabei der ihnen verbliebenen sozialen und kulturellen Ressourcen bedienen. Zum anderen ähneln die von ihnen verfolgten Strategien häufig jenen der sogenannten Arbeitsmigranten. Und schliesslich sind die Grenzen zwischen den verschiedenen Status (Vertriebene im eigenen Land, Flüchtlinge, Arbeitsmigranten) durchlässig: Durch die Anpassung an die sich ständig wandelnden Umstände und durch bewusstes „Austauschen von Etiketten" kann ein und dieselbe Person nacheinander oder gleichzeitig mehreren Kategorien angehören.

Gemäss transnationaler Perspektive wird die Gesamtheit der betroffenen Orte als System oder Einheit untersucht. In der Tat ist die Aufsplitterung familiärer Gruppen häufig das Ergebnis einer Strategie zur Ressourcendiversifizierung und Risikostreuung und geht somit nicht zwangsläufig mit einer Schwächung der Beziehungen einher. Wie bereits erwähnt, entspricht dies den Grundsätzen der Neuen Ökonomie der Arbeitsmigration (new economics of labor migration, NELM ${ }^{34}$. Während die neoklassische Lehre das Individuum und dessen rationelle Entscheidungen in den Mittelpunkt stellt, betrachtet die NELM den Haushalt - in seinen verschiedenen soziokulturellen Erscheinungsformen - als Grundeinheit der Entscheidungsfindung. Statt Gewinnmaximierung stehen hier die Risikoverminderung und der Zugang zu einer grösseren Ressourcenvielfalt im Vordergrund. Unter diesem Blickwinkel spielen die Remittances eine zentrale Rolle, denn sie ermöglichen eine Diversifizierung und Erhöhung des Haushaltseinkommens und zeigen Wege auf, um lokale ökonomische Schwierigkeiten und Hindernisse - namentlich der erschwerte Zugang zu Kredit - zu überwinden und zu umgehen. Durch die geplante geografische Zerstreuung der Mitglieder eines Haushalts lässt sich die Migration in diesem Fall als Strategie zur Diversifizierung des sozialen und ökonomischen Kapitals betrachten, in anderen Worten als Versicherung gegen eine nie auszuschliessende Verschlechterung der Lebensbedingungen im Herkunftsland oder eine veritable livelihood strategy ${ }^{35}$. Inwiefern aber können diese Überlegungen zu Transnationalismus, Neuer Ökonomie der Arbeitsmigration und Subsistenzstrategien zur Klärung der Beziehungen zwischen Migration und Entwicklung beitragen? Ihr Verdienst ist es insbesondere, die Akzeptanz des Gedankens zu fördern, dass die erfolgreiche wirtschaftliche und soziale Integration eines Migranten im Gastland nicht zwangsläufig mit einem Bruch der Beziehungen zu seiner Herkunftsgesellschaft einhergeht. Migranten können sich komplementär und gleichzeitig in zwei oder

34 O. Stark and D.E. Bloom, „The New Economics of Labor Migration“, American Economic Review, Bd. 75, Nr. 2, 1985, S. 173-178; siehe auch D.S. Massey et al., op. cit., S. 440-442.

35 In der englischsprachigen Entwicklungsliteratur wird livelihood als Gesamtheit der (materiellen und sozialen) Mittel verstanden, mit denen die Bedürfnisse gedeckt werden. Dazu zählen namentlich Beschäftigung, Institutionen sowie zwischenmenschliche und interfamiliäre Beziehungen. Heute ist häufig die Rede von natürlichem, sozialem, humanem, physischem, ökonomischem oder finanziellem Kapital, wobei diese Begriffe zuweilen mit einem Mangel an Präzision behaftet sind. Livelihood strategy bezeichnet demnach die bewussten Entscheidungen, verschiedene Ressourcen und Tätigkeiten miteinander zu kombinieren. 
mehr Gesellschaften engagieren, das heisst, ein integrierter Migrant kann durchaus weiterhin als Akteur der Entwicklung seiner Herkunftsgesellschaft tätig und gleichzeitig am politischen Leben im Gastland teilnehmen. Entgegen der Auffassung der Modernisierungstheorie muss man nicht zwingend in seine Herkunftsgesellschaft zurückkehren, um aktiv zu ihrer Entwicklung beizutragen, und entgegen der Meinung der dependantistas ist Migration nicht gleichbedeutend mit Handlungsunvermögen. Diese Sichtweise widerspricht auch der Überzeugung mancher Forschender, wonach Migrantinnen und Migranten ihre Beziehungen zu ihrem Herkunftsmilieu nach und nach aufgeben. Im Gegenteil : Der Transnationalismus macht deutlich, dass die ersten Migranten mit ihren Erfahrungen und Kontakten den Weg der „zweiten Migrantengeneration“ vorzeichnen, indem sie die Letzteren mit Informationen über Beschäftigungsmöglichkeiten sowie über Anlaufstellen für Unterstützung, Wohnmöglichkeiten, Identitätspapiere oder Aufenthaltsgenehmigungen versorgen. Auf diese Weise werden Faktoren berücksichtigt, die die Dauerhaftigkeit der Migration stärken, wie etwa eine Relaisstrategie: Die Mitglieder einer Familie wandern in geplanter Abfolge aus, um im Ausland eine Erwerbstätigkeit zu suchen, von der die ganze Gemeinschaft profitiert, das heisst, die Rückkehr eines Haushaltsmitglieds fällt häufig zeitlich zusammen mit dem Weggang eines anderen ${ }^{36}$. Kurzum: Sozialen Netzen wird hier eine wichtige Rolle zuerkannt, denn sie erleichtern den Informationsfluss zwischen Migranten und Nicht-Migranten und können Aufschluss geben über die massiven Summen, die Migranten in ihre Heimat überweisen.

\section{Rücküberweisungen und Entwicklung}

Die Diskussionen über die Wirkungen der Remittances im Kampf gegen die Armut und insbesondere über ihren Beitrag an Wirtschaft und Gesellschaft der Herkunftsländer widerspiegeln zu einem grossen Teil die Debatte über die Beziehungen zwischen Migration und Entwicklung. Der transnationale Ansatz und die Neue Ökonomie der Arbeitsmigration vermögen die sozialen Bindungen, die den bedeutenden Rücküberweisungen der Migranten zugrunde liegen, weitaus deutlicher darzulegen als die Modernisierungstheorie, welche den Migranten hauptsächlich nach ihrer Rückkehr eine Schlüsselrolle bei der Entwicklung ihrer Herkunftsregion zugesteht, oder die Abhängigkeitstheorie, die die internationale Migration ausschliesslich als Zeichen des Niedergangs der Volkswirtschaften und Gesellschaften des Südens betrachtet.

Wie bereits erwähnt (siehe Tabelle 1), geht es hierbei um enorme Beträge, die jene der APD bei Weitem übersteigen. Nach Auffassung zahlreicher Forschender - die von den Entwicklungsinstitutionen aufgegriffen wurde - sind Remittances als wirksame Überlebensstrategie und unverzichtbares Mittel zur Armutsbekämpfung sowie in Abhängigkeit des sozialen Zusammenhalts zu betrachten, der von diesem gigantischen Vermögenskreislauf reproduziert wird ${ }^{37}$.

36 H. de Haas, Migration and Development, op. cit., S. 19, 22; D.S. Massey et al., op. cit., S. 460-462. Zur Relaisstrategie afghanischer Migranten im Iran siehe beispielsweise A. Monsutti, Guerres et migrations, op. cit., S. 207, 209 und 225.

37 Die Literatur über Rücküberweisungen von Migranten, Armutsbekämpfung und Entwicklung ist sehr umfangreich und umfasst zahlreiche Berichte der Weltbank, der Internationalen Organisation für 
Vor einer allzu gutgläubigen Sicht sei aber gewarnt. Einigen Autoren zufolge werden die von Migranten überwiesenen Gelder nicht in produktive Tätigkeiten investiert, sondern für ostentative Ausgaben wie für den Erwerb von Konsumoder Luxusgütern oder Immobilien verwendet. Sie trügen somit kaum zur wirtschaftlichen Entwicklung der Herkunftsländer bei, und anstatt die Produktion zu stärken und Arbeitsplätze zu schaffen, förderten sie inflationäre Tendenzen und zementierten die Abhängigkeit vom Ausland. Da es nicht die Ärmsten seien, die auswanderten und von Rücküberweisungen profitierten, würden bestehende Ungleichheiten noch akzentuiert. Ausserdem dienten gewisse informelle Transfersysteme zur Geldwäscherei und ermöglichten es Terrornetzwerken, ihre Gelder diskret in Umlauf zu bringen ${ }^{38}$. Einige der wenigen positiven Auswirkungen der Rücküberweisungen von Migranten sei es, dass sie mehr Kindern den Schulbesuch erlaubten ${ }^{39}$.

Umstritten ist auch die Rolle der Rücküberweisungen in Konfliktsituationen, auch wenn diese Frage weitaus weniger Beachtung fand ${ }^{40}$. Zweifellos können Gelder von Migranten in Konfliktsituationen oder in einem noch ungefestigten Frieden zur Belebung der Wirtschaftstätigkeit beitragen ${ }^{41}$. Dennoch besteht immer die Gefahr, dass sie von am Konflikt beteiligten Gruppen vereinnahmt werden und so innere Auseinandersetzung schüren ${ }^{42}$. In Situationen mit schwachen staatlichen Strukturen gelänge es gewissen Führungspersönlichkeiten oder politischen Gruppierungen, sich Ressourcen anzueignen und die Kontrolle über Verteilkanäle von Gütern und Dienstleistungen zu erlangen, um diese nach eigenem Ermessen zu verteilen und ihren politischen Einfluss auszubauen.

Tabelle 2 fasst die Argumente beider Seiten zusammen:

Migration und verschiedener nationaler Entwicklungsagenturen wie die britische DFID, darunter: Committee on Payment and Settlement Systems and World Bank, General Principles for International Remittance Services, Basel, Bank for International Settlements; Washington, DC, World Bank, March 2006. Erwähnt seien ferner die Sondernummer The Migration-Development Nexus von International Migration, Bd. 40, Nr. 5, 2002; sowie ein Beitrag, der die ökonomischen Aspekte beleuchtet: R.H. Adams and J. Page, „Do International Migration and Remittances Reduce Poverty in Developing Countries?", World Development, Bd. 22, Nr. 10, 2005, S. 1645-1669. Siehe auch verschiedene Texte von R. Ballard auf der Website des Centre for Applied South Asian Studies, University of Manchester, <http://www.art.man.ac.uk/CASAS/pages/papers.htm>.

38 Diesem Vorwurf sieht sich häufig das hawâla-System ausgesetzt, obwohl sich dieses für die Afghanen als äusserst wirksame Überlebensstrategie bewährt hat (siehe den Beitrag des Autors zu diesem Thema im vorliegenden Band).

39 Siehe unter anderem M. Kearney, op. cit., S. 346-347; oder M.-A. Pérouse de Montclos, „Réseaux financiers, diasporas et hawilad: le rôle clé de la péninsule Arabique entre l'Occident et la Somalie“, in E. Grégoire et J. Schmitz (dir.), Afrique noire et monde arabe: continuités et ruptures, Paris, Aube; IRD, 2000, S. 114-115.

40 Siehe P. Collier, Economic Causes of Civil Conflict and Their Implications for Policy, Washington, DC, World Bank, 2000, sowie seine Kritiker, R. Marchal et Ch. Messiant, „De l'avidité des rebelles: l'analyse économique de la guerre civile selon Paul Collier", Critique internationale, Bd. 16, Juli 2002, S. 59-69. Diese Autoren konzentrieren sich indessen eher auf die Rolle bewaffneter Gruppierungen als auf jene der Diasporen.

41 Siehe hierzu einen Bericht, der im Rahmen des Security-Development Nexus Programme der International Peace Academy veröffentlicht wurde: P. Weiss Fagen and M.N. Bump, Remittances in Conflict and Crises: How Remittances Sustain Livelihoods in War, Crises, and Transitions to Peace, Washington, DC, International Peace Academy, February 2006.

42 So stehen beispielsweise die Befreiungstiger von Eelam Tamoul (besser bekannt unter der Abkürzung LTTE), die Kurdische Arbeiterpartei (PKK) oder die Befreiungsarmee des Kosovo (UÇK) im Verdacht, sich zum Teil über Gelder von Migranten zu finanzieren. 
Tabelle 2: Positive und negative Auswirkungen von Remittances auf die Entwicklung

\section{Positiv}

- Wirksame Überlebensstrategie

- Diversifizierung der Einkommensquellen - Migration als Sozialversicherung

- Verringerung der Armut im Herkunftsland

- Stärkung der Kaufkraft, Multiplikatorwirkung für die gesamte Wirtschaft

- Potenziell produktive Investitionen (ca. 20 Prozent der überwiesenen Gelder)

- Gründung von Kleinunternehmen

- Ausgleich des Einkommensverlustes aufgrund von Konflikten

- Gemeinschaftliche Entwicklung dank Migrantenvereinigungen

- Bildungsförderung (unter den im Herkunftsland gebliebenen Angehörigen der Migranten); Wissensvermittlung und Rückkehr von Migranten, die sich im Ausland Kompetenzen angeeignet haben (brain gain); positive Auswirkungen auf die Gesundheit, Sensibilisierung für Pflege usw.

- Förderung des Friedens, der Menschenrechte und der Demokratie (social remittances)

- Soziale Dimension: Zusammenhalt innerhalb von Solidaritätsgruppen trotz geografischer Entfernung, Errichtung transnationaler Netze

Negativ

- „Ausbluten“ der Gesellschaft, Abfluss von Arbeitskräften (wiegt besonders schwer in agrarisch geprägten Volkswirtschaften)

- Herausbildung einer Abhängigkeitskultur

- Investitionen in Immobilien und Konsumgüter, Anstieg der Lebenskosten (insbesondere der Mieten)

- Private Dimension der Transfers, geringer Nutzen für die Gemeinschaft

- Verschärfung der Ungleichheiten: Die ärmsten Haushalte können es sich nicht leisten, eines ihrer Mitglieder ins Ausland zu schicken; Vertiefung der Kluft zwischen Reich und Arm

- Wegzug von qualifizierten Personen (brain drain) und Nichteingliederung von Migranten, die mit neuen, im Ausland erworbenen Kompetenzen zurückkehren (brain waste)

- Entgegen der Subsidiaritätstheorie der Weltbank schicken gut ausgebildete Migranten nur wenig Geld in die Heimat zurück.

- Nährboden für Korruption

- Finanzierung von bewaffneten Gruppierungen, Missbrauch durch internationale Terrorismusnetze

- Waschen von Geldern krimineller Herkunft

- Gefahr einer Stärkung der Schlepperei (human trafficking) bzw. von Verbindungen zu Schlepperorganisationen

Diese Aufzählung macht deutlich, dass finanzielle und soziale Transfers von Migranten durchaus ein Potenzial für die Entwicklung der Herkunftsregionen bergen, aber auch mit Gefahren verbunden sind. In Anbetracht dieser stark divergierenden Argumente und der Verschiedenartigkeit der Fälle wäre eine abschliessende Stellungnahme wenig sinnvoll.

\section{Fazit und Ausblick}

Die obigen Ausführungen haben gezeigt, dass die Beziehungen zwischen Migration und Entwicklung komplex sind und sich jeder einseitigen Betrachtungsweise - sei diese optimistisch oder pessimistisch - entziehen. Gefordert ist vielmehr ein pluralistischer und flexibler Ansatz, der der zeitlichen und räumlichen Verschiedenartigkeit der Situationen gerecht wird. Der heute überwiegende institutionelle Optimismus vernachlässigt die theoretischen Debatten, die die Sozialwissenschaften seit den 1950er-Jahren beherrscht haben. Ausserdem begnügt er sich häufig mit Absichtserklärungen und führt nur selten zu konkreten politischen Massnahmen. Die historische Kurzsichtigkeit sowie die Kluft zwischen Diskurs und Praxis sind frappierend. 
Die transnationale Sichtweise macht deutlich, dass Migrantinnen und Migranten im Laufe der Zeit vielfältige Beziehungen knüpfen und häufig in mehreren Gesellschaften gleichzeitig als Akteure auftreten. Um die Komplexität der Phänomene erfassen zu können, müssen die Kategorisierung der Migranten überwunden und die Nichtlinearität ihrer Laufbahnen anerkannt werden: Flüchtlinge können zu Unternehmern werden und Geld in ihre Heimat schicken, genauso wie Studierende während ihres Aufenthalts im Ausland Ersparnisse bilden und Rücküberweisungen an ihre Familien tätigen können. Dass Migranten und Flüchtlinge so zur wirtschaftlichen Entwicklung ihrer Herkunftsländer beitragen können, lässt sich nicht von der Hand weisen. Aber Migration und Entwicklung verhalten sich nicht umgekehrt proportional zueinander. Die bisherige empirische Forschung hat gezeigt, dass die Vielfalt der Fälle nur schwer auf einige grundlegende Muster reduziert werden kann. Ein eindeutiger und absoluter Bezug zwischen Rücküberweisungen und Armutsverringerung oder zwischen Wirtschaftswachstum und Eindämmung der Migrationsströme wurde nicht nachgewiesen. Ausserdem scheinen Reichtumsunterschiede häufiger eine entscheidende Rolle zu spielen als die Armut an sich. Migration ist nicht zwangsläufig eine verzweifelte Flucht vor Not und Gewalt, sondern häufig Ausdruck einer bewusst angestrebten geografischen, sozialen, wirtschaftlichen und politischen Diversifizierung mit dem Ziel, lokale Sachzwänge zu überwinden. Die räumliche Zerstreuung der Mitglieder eines Haushalts ist demnach als eine Art Versicherung gegen eine Verschlechterung der Lebensbedingungen in der Heimat zu betrachten.

Dieser Blickwinkel deckt die Gesamtheit aller möglichen Fälle ab, angefangen bei der rein freiwilligen Auswanderung bis hin zum Exodus von Menschen, die vor Bomben fliehen. Mobilität ist häufig eine bewusst gewählte Option, die sich in den Lebenslauf der Menschen einfügt und zu einer Neudefinition der Beziehungen zwischen Generationen und Geschlechtern beiträgt. Junge Männer beispielsweise können so zu ihrer Männlichkeit finden und ihren Erwachsenenstatus festigen ${ }^{43}$. Zahlreiche Frauen wiederum wandern aus eigenem Entschluss aus oder haben als Ehefrauen eines Auswanderers die Chance, neue Verantwortungen zu übernehmen, sei es im Haushalt, in der Führung eines Landwirtschaftsbetriebs oder ganz allgemein in Entscheidungsprozessen, die die ganze Familie betreffen. Die globale wirtschaftliche Umstrukturierung führt zu einer neuen internationalen Arbeitsteilung. Frauen wie Jugendlichen - seien sie selbst Migranten oder nicht - eröffnet sich damit die Möglichkeit, gegen althergebrachte hierarchische Ordnungen anzukämpfen und neue Freiräume zu erobern ${ }^{44}$.

Aus dieser Sicht wird Migration als ein seit jeher konstituierendes Element menschlicher Gesellschaften erörtert. Das gegenwärtige Interesse an den Remittances ist insofern ermutigend, als es dazu beiträgt, den Nutzen der Mobilität anzuerkennen. Allerdings besteht die Gefahr einer Fehleinschätzung und einer politischen Instrumentalisierung. Rücküberweisungen von Migranten tragen

43 Siehe beispielsweise F. Osella and C. Osella, „Migration, Money and Masculinity in Kerala“, Journal of the Royal Anthropological Institute, Bd. 6, 2000, S. 117-133; A. Monsutti, „Migration as a Rite of Passage: Young Afghans Building Masculinity and Adulthood in Iran“, Iranian Studies, Bd. 40, Nr. 2, 2007, S. 167-185.

44 Die Literatur über die Neudefinition der Gender-Beziehungen im Zuge der Migration ist sehr umfangreich. Erwähnt sei an dieser Stelle das bereits zitierte Werk von B. Ehrenreich und A.R. Hochschild. 
ohne Zweifel dazu bei, die Risiken der begünstigten Haushalte zu verringern und ihre Einkünfte zu erhöhen. Dies bedeutet jedoch nicht, dass sie wesentlich und gesamthaft in den Entwicklungsländern die Armut senken oder das BIP erhöhen. Die strukturellen Probleme der Unterentwicklung werden durch sie nicht gelöst - dies ist die Aufgabe der öffentlichen Entwicklungshilfe. Angesichts dessen ist dem prävalenten Optimismus mit Zurückhaltung zu begegnen und bei der Betrachtung der Beziehungen zwischen Migration und Entwicklung ein pluralistischer Ansatz zu bevorzugen. Voreilige Verallgemeinerungen sind zwecklos, denn die Beiträge der Migranten an ihre Herkunftsgesellschaften variieren von Fall zu Fall.

Das Hohelied, das derzeit auf die internationale Migration und auf die Rücküberweisungen als neues Patentrezept gegen die Unterentwicklung der Länder des Südens gesungen wird, ist zumindest mit einem verdächtigen Mangel an historischer und theoretischer Perspektive behaftet. Ausserdem birgt es einen inneren Widerspruch, denn die Migration wird zum Grundsatz ihrer Verneinung erhoben: Einerseits werden die Migrantinnen und Migranten als ,verkannte Helden der internationalen Entwicklung" gefeiert, und andererseits wird von der Entwicklung erwartet, dass sie die globalen Migrationsströme eindämmt. Ohne nun mit einer Provokation schliessen zu wollen, sei mit Thomas Faist und anderen Autoren die Frage gestellt, ob die Diskurse, die die Beziehungen zwischen Migration und Entwicklung betonen, nicht letzten Endes dazu dienen sollen, den im Norden wie im Süden wachsenden politischen Willen zur Kontrolle und Eindämmung der Migrationsströme zu legitimieren ${ }^{45}$. Die Frage mag unverschämt und störend sein, aber sie ist es allemal wert, gestellt zu werden!

\section{Bibliographie (Auswahl)}

Adams, R.H. and J. Page, „Do International Migration and Remittances Reduce Poverty in Developing Countries ?", World Development, Bd. 22, Nr. 10, 2005, S. 1645-1669.

Ballard, R., „Migration, Remittances, Economic Growth and Poverty Reduction: Reflections on Some South Asian Developments“, in T. Siddiqui (ed.), Migration and Development: Pro-Poor Policy Choices, Dhaka, The University Press Ltd, 2005, S. 333-358.

Faist, Th., „Migrants as Transnational Development Agents: An Inquiry into the Newest Round of the Migration-Development Nexus“, Population, Space and Place, Bd. 14, 2008, S. 21-42.

Global Commission on International Migration, Migration in an Interconnected World: New Directions for Action, Geneva, Global Commission on International Migration, October 2005.

Gosh, B., Migrants' Remittances and Development: Myth, Rhetoric and Realities, Geneva, International Organization for Migration; The Hague, The Hague Process on Refugees and Migration, 2006.

Haas, H. de, Engaging Diasporas: How Governments and Development Agencies Can Support Involvement in the Development of Origin Countries, Oxford, International Migration Institute, University of Oxford, 2006.

Haas, H. de, Migration and Development: A Theoretical Perspective, Oxford, International Migration Institute, University of Oxford, 2007.

Haas, H. de, Turning the Tide? Why „Development Instead of Migration“ Policies are Bound to Fail, Oxford, International Migration Institute, University of Oxford, 2006.

Kapur, D., Remittances: The New Development Mantra?, G-24 Discussion Paper Series, Nr.29, New York; Geneva, United Nations Conference on Trade and Development, 2004.

Kearney, M., „From the Invisible Hand to Visible Feet: Anthropological Studies of Migration and Development", Annual Review of Anthropology, Bd. 15, 1986, S. 331-361.

45 Th. Faist, „Migrants as Transnational Development Agents“, op. cit., S. 38. 
Massey, D.S., J. Arango, G. Hugo, A. Kouaouci, A. Pellegrino and J.E. Taylor, „Theories of International Migration: A Review and Appraisal“, Population and Development Review, Bd.19, Nr.3, 1993, S. 431-466,

Nyberg-Sørensen, N., N. Van Hear and P. Engberg-Pedersen, „The Migration-Development Nexus: Evidence and Policy Options“, International Migration, Bd. 40, Nr. 5, 2002, S. 49-73.

Nyberg-Sørensen, N., N. Van Hear and P. Engberg-Pedersen, „The Migration-Development Nexus: Evidence and Policy Options - State-of-the-art Overview“, International Migration, Bd. 40, Nr. 5, 2002, S. 3-47.

Pessar, P. and S.J. Mahler, „Transnational Migration: Bringing Gender In“, International Migration Review, Bd. 37, Nr. 3, 2003, S. 812-846.

Ratha, D., „Workers' Remittances: An Important and Stable Source of External Development Finance“, Global Development Finance 2003, Washington, DC, World Bank, 2003, S. 157-175.

Vertovec, S., „Conceiving and Researching Transnationalism“, Ethnic and Racial Studies, Bd. 22, Nr. 2, 1999, S. 447-462.

Weiss Fagen, P. and M.N. Bump, Remittances in Conflict and Crises: How Remittances Sustain Livelihoods in War, Crises, and Transitions to Peace, Washington, DC, International Peace Academy, February 2006. 